\title{
Enhanced non-volatile memory characteristics with quattro-layer graphene nanoplatelets vs. 2.85-nm Si nanoparticles with asymmetric $\mathrm{Al}_{2} \mathrm{O}_{3} / \mathrm{HfO}_{2}$ tunnel oxide
}

\author{
Nazek El-Atab ${ }^{1 *}$, Berk Berkan Turgut ${ }^{2}$, Ali K Okyay ${ }^{2,3,4}$, Munir Nayfeh ${ }^{5}$ and Ammar Nayfeh ${ }^{1}$
}

\begin{abstract}
In this work, we demonstrate a non-volatile metal-oxide semiconductor (MOS) memory with Quattro-layer graphene nanoplatelets as charge storage layer with asymmetric $\mathrm{Al}_{2} \mathrm{O}_{3} / \mathrm{HfO}_{2}$ tunnel oxide and we compare it to the same memory structure with 2.85-nm Si nanoparticles charge trapping layer. The results show that graphene nanoplatelets with $\mathrm{Al}_{2} \mathrm{O}_{3} / \mathrm{HfO}_{2}$ tunnel oxide allow for larger memory windows at the same operating voltages, enhanced retention, and endurance characteristics. The measurements are further confirmed by plotting the energy band diagram of the structures, calculating the quantum tunneling probabilities, and analyzing the charge transport mechanism. Also, the required program time of the memory with ultra-thin asymmetric $\mathrm{Al}_{2} \mathrm{O}_{3} / \mathrm{HfO}_{2}$ tunnel oxide with graphene nanoplatelets storage layer is calculated under Fowler-Nordheim tunneling regime and found to be 4.1 ns making it the fastest fully programmed MOS memory due to the observed pure electrons storage in the graphene nanoplatelets. With Si nanoparticles, however, the program time is larger due to the mixed charge storage. The results confirm that band-engineering of both tunnel oxide and charge trapping layer is required to enhance the current non-volatile memory characteristics.
\end{abstract}

Keywords: Charge trapping memory devices; Graphene nanoplatelets; Silicon nanoparticles; Aluminum oxide; Atomic layer deposition; Retention time; Program time

\section{Background}

The demand for low-power, high-speed, and highdensity non-volatile memory devices has increased drastically over the past decade due to the growing market of consumer electronics. However, current flash memory devices are expected to face two major challenges in the near future: density and voltage scaling. The density of the memory is related to the gate length scaling which is constrained by the gate stack, precisely, the tunnel oxide thickness. In fact, the gate length is required to be adequate with the gate stack in order to maintain a good gate control and to avoid short channel effects. However, in conventional flash memories, the tunnel oxide thickness has a lower limit of 6-8 nm (depending on NOR or

\footnotetext{
* Correspondence: nelatab@masdar.ac.ae

${ }^{1}$ Institute Center for Microsystems-iMicro, EECS, Masdar Institute of Science and Technology Abu Dhabi, Abu Dhabi, United Arab Emirates

Full list of author information is available at the end of the article
}

NAND structure) in order to avoid back-tunneling and thus leakage of charges which destroys the necessary retention characteristic of the memory ( $>10$ years). The second problem which needs to be solved is the high program and erase operating voltages. Once again, the limitation to operating voltage scaling is the inability to reduce gate stack thickness. In addition to the trade-off relationship between tunnel oxide thickness and retention characteristic of the memory where the retention of charges is exponentially degraded as the tunnel oxide thickness is scaled down, there exists another trade-off relationship between the tunnel oxide thickness and the resulting program time, where a thicker tunnel oxide causes the extension of the time needed for the charges to be transported from the channel to the charge trapping layer and vice-versa. Therefore, it is imperative to find novel structures and materials to be incorporated in 
the memory cells which would allow tunnel oxide and voltage scaling.

Since its first discovery in 2004 [1], graphene has attracted major attention and is currently considered as a promising material in next-generation information-processing devices due to its outstanding electronic properties [2]. However, the sole use of pristine graphene as the charge storage layer is not enough to enhance the current non-volatile memory characteristics [3]. The choice of the tunnel oxide material of the memory has a significant impact on the memory performance [4]. On the other hand, Si-nanoparticle-based memory has been extensively investigated, and on the industry side, it was considered as a viable memory system due to the larger retention time, lower power consumption, and faster operation than conventional polysilicon-based flash memory $[5,6]$. Freescale demonstrated a 4-Mbit flash memory device as early as 2003 and has most recently (2006) demonstrated a 24-Mbit flash memory device using Si nanoparticle materials.

In this work, we demonstrate a non-volatile metaloxide semiconductor (MOS) memory with Quattrolayer graphene-nanoplatelets as charge storage layer with asymmetric $\mathrm{Al}_{2} \mathrm{O}_{3} / \mathrm{HfO}_{2}$ tunnel oxide and we compare it to the same memory structure with $2.85-\mathrm{nm} \mathrm{Si}$ nanoparticles charge trapping layer. TEM images, electrical characterization, construction of the energy band diagrams of the MOS memory devices, and quantum mechanical calculations are provided to confirm the importance of the band-engineering of both tunnel oxide and charge trapping layer of non-volatile memory devices. In addition, the results show that MOS memory devices with Quattro-layer graphene-nanoplatelets as charge storage layer with asymmetric $\mathrm{Al}_{2} \mathrm{O}_{3} / \mathrm{HfO}_{2}$ tunnel oxide has potential in future low-power and fast non-volatile memory devices.

\section{Methods}

The MOS memory devices are fabricated on lowresistivity n-type $\mathrm{Si}(111)$ substrate (Antimony-doped, $15-20 \mathrm{~m} \Omega / \mathrm{cm})$. A $4-\mathrm{nm} \mathrm{Al}_{2} \mathrm{O}_{3}$ tunnel oxide is first deposited by thermal atomic layer deposition (ALD) at 250 ${ }^{\circ} \mathrm{C}$ using a Cambridge Nanotech Savannah-100 atomic layer deposition system followed by $1.1 \mathrm{~nm} \mathrm{HfO}_{2}$ deposited by plasma-assisted ALD (PA-ALD) at $195{ }^{\circ} \mathrm{C}$ using an Oxford FlexAL system. Next, the sample is placed on a hot plate at $110{ }^{\circ} \mathrm{C}$, and $2-2.5 \mathrm{ml}$ of pristine graphene nanoplatelets (Quattro-layer, $0.05 \mathrm{mg} / \mathrm{ml}$ ) with an average size of $4.4 \mathrm{~nm}$ (see Additional file 1: Figure S1) are drop-casted on the sample. Then, $1.1 \mathrm{~nm} \mathrm{HfO}_{2}$ is deposited by PA-ALD at $195{ }^{\circ} \mathrm{C}$ followed by $6.5-\mathrm{nm} \mathrm{Al}_{2} \mathrm{O}_{3}$ blocking oxide deposited at $250{ }^{\circ} \mathrm{C}$ by ALD. Finally, a shadow mask with feature size down to $10 \mu \mathrm{m}$ is used to pattern the 400-nm Al gate contact deposited by e-beam evaporation. The same process is repeated to fabricate the MOS memory with 2.85-nm Si nanoparticles [7] (see Additional file 1: Figure S2), where Si nanoparticles are spin-coated on the sample at a speed of $2000 \mathrm{rpm}$ and acceleration of $500 \mathrm{rpm} / \mathrm{s}$ for $45 \mathrm{~s}$. TEM cross-section of the MOS memory with graphene nanoplatelets is shown in Fig. 1a where an interfacial $1 \mathrm{~nm} \mathrm{SiO}_{2}$ is observed (see Additional file 1: Figure S3 also). A cross-section illustration of the fabricated memory with graphene nanoplatelets is also shown in Fig. 1b.

The electrical measurements are done using an Agilent B1505A semiconductor device analyzer.

\section{Results and Discussion}

To analyze the memory performance, high-frequency $(1 \mathrm{MHz}) \mathrm{C}-\mathrm{V}_{\text {gate }}$ measurements are conducted. The gate voltage is first swept from -7 to $7 \mathrm{~V}$ which resulted in the erased-state, then from 7 to $-7 \mathrm{~V}$ resulting in the programmed state. The obtained memory hysteresis is $3.1 \mathrm{~V}$ with graphene nanoplatelets while $2.9 \mathrm{~V}$ with Si nanoparticles. The measurements are repeated at different gate voltages as shown in Fig. 2a, b for the memory with graphene nanoplatelets and Si nanoparticles, respectively. It is observed that the memory with $\mathrm{Si}$ nanoparticles is programmed by storing electrons and erased by storing holes as shown by the positive and negative shifts in the programmed and erased states of Fig. 2b, respectively. It is also shown in Fig. $2 b$ that additional charging is due to holes at large erasing voltages of $-8 \mathrm{~V}$ corresponding to an electric field across the tunnel oxide $\mathrm{Al}_{2} \mathrm{O}_{3}\left(E_{\text {ox }}\right)$ of $10.6 \mathrm{MV} / \mathrm{cm}$ whereas the memory with graphene nanoplatelets is programmed by storing electrons and erased through backtunneling of electrons which is shown by the shift of the programmed state in Fig. 2a. The threshold voltage $\left(V_{\mathrm{t}}\right)$ shift achieved with graphene nanoplatelets is higher than the $V_{\mathrm{t}}$ shift achieved with Si nanoparticles at different gate voltages as shown in Fig. 2c.

The memory endurance characteristic is studied by plotting the $V_{\mathrm{t}}$ shift vs. the number of program/erase cycles at 8/-8 V as depicted in Fig. 2d. Non-volatile memories can be programmed/erased frequently at the expense of introducing permanent gate-oxide damage such as the trapping of electrons/holes in the available trapping states in the oxide [8]. These trapped charges change the injection fields and, thus, the amount of charge transferred to and from the charge storage layer during programming. The lower endurance with $\mathrm{Si}$ nanoparticles after $10^{4}$ cycles (33.3\% degradation) than the memory endurance with graphene $(20 \%)$ can be due to two reasons: first, the larger accumulation capacitance $\left(C_{\text {acc }}\right)$ of the memory with $\mathrm{Si}$ nanoparticles and the similar $\Delta V_{\mathrm{t}}$ at $8 \mathrm{~V}$ results in a larger trapped charge density $\left(\Delta Q=C_{\text {acc }} \times \Delta V_{\mathrm{t}}\right)$ in the Si nanoparticles $\left(\Delta \mathrm{Q}\right.$ in Si nanoparticles $\sim 8.3 \times 10^{13} \mathrm{~cm}^{-2}>\Delta Q$ in graphene nanoplatelets $\sim 7.3 \times 10^{13} \mathrm{~cm}^{-2}$ ) which means that more charges are tunneling through the tunnel oxide 

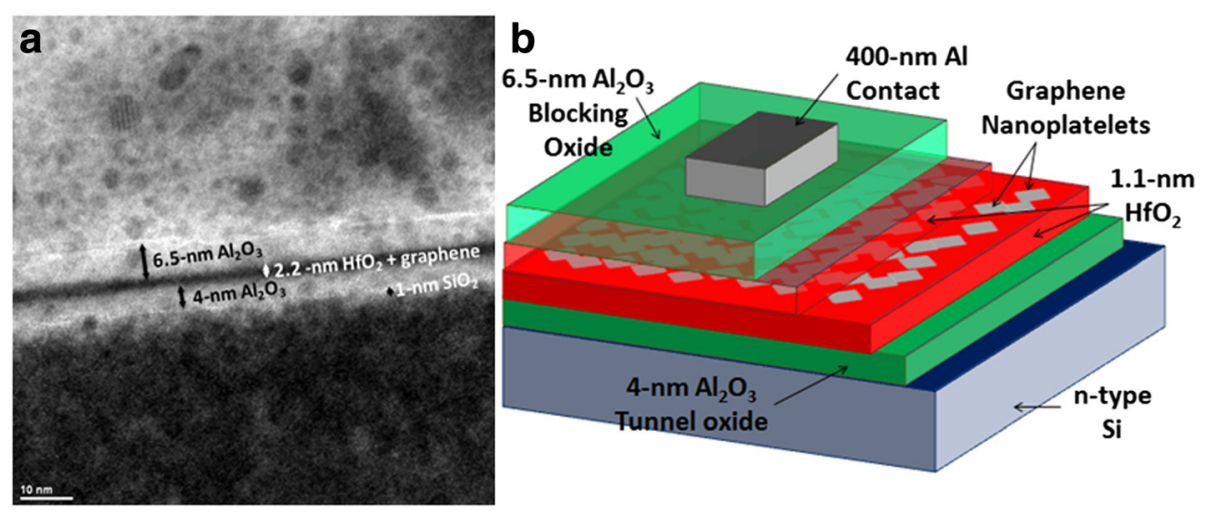

Fig. 1 Fabricated memory devices; a TEM cross-section of the memory with graphene nanoplatelets. b Cross-section illustration of the fabricated memory cells with graphene nanoplatelets. The memory with Si nanoparticles has the same cross-section illustration

of the memory with Si nanoparticles which might increase the degradation of the oxide. Second, with Si nanoparticles, both electrons and holes are tunneling through the tunnel oxide during program/erase cycles. As a result, both electrons and holes will be trapped in the available trapping states in the oxide further degrading the endurance characteristic with respect to the memory with graphene nanoplatelets where only electrons are tunneling.

Moreover, the retention of the memory cells is characterized by first programming/erasing the memory at $8 /-8$ $\mathrm{V}$ and observing the change in $V_{\mathrm{t}}$ shift in time as shown in Fig. 3a, b for the memory devices with graphene nanoplatelets and Si nanoparticles, respectively. The enhanced retention with graphene ( $28.8 \%$ loss of initial stored charge) at 10 years with respect to the retention of the memory with Si nanoparticles $(35.5 \%)$ is due to the larger electron affinity of graphene [9] (4.6 eV) than 2.85-nm Si nanoparticles [10] $(2.9 \mathrm{eV})$ which increases the conduction band offset (CBO) between charge storage layer and tunnel oxide, and therefore exponentially reduces the backtunneling of electrons.

The energy band diagrams of the memory structures with graphene and Si nanoparticles are plotted in Fig. 4a, b, respectively [11-22]. The smaller $\mathrm{CBO}$ than valence band offset $(\mathrm{VBO})$ between the substrate and $\mathrm{Al}_{2} \mathrm{O}_{3}$ confirms the observed electrons storage during programming of both memories. In order to analyze the charge emission mechanism, the electric field across $\mathrm{Al}_{2} \mathrm{O}_{3}$ is calculated using Gauss's law [17], and the $V_{\mathrm{t}}$ shift vs. $\left(E_{\mathrm{ox}}\right)^{2}$ is plotted in Fig. $5 \mathrm{a}$, and the linear region suggests that phonon-assisted
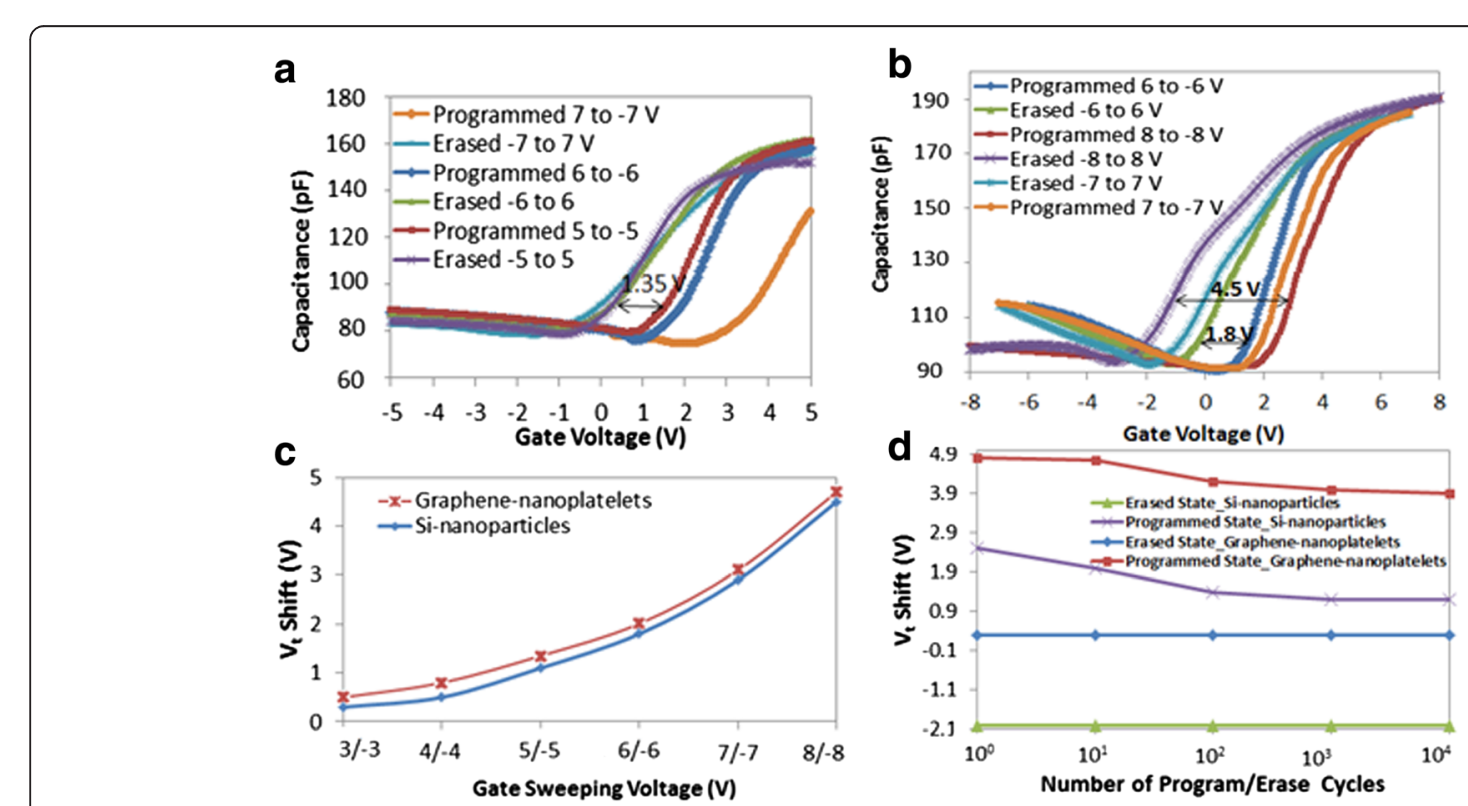

Fig. 2 Electrical characterization of the memory devices; a High-frequency $(1 \mathrm{MHz}) \mathrm{C}-\mathrm{V}$ measurements of the memory with graphene nanoplatelets. b High-frequency ( $1 \mathrm{MHz}$ ) C-V measurements of the memory with Si nanoparticles. c Plot showing the measured $V_{t}$ shifts at different gate sweeping voltages. $\mathbf{d}$ Endurance characteristic of the memory devices programmed/erased at $8 /-8 \mathrm{~V}$ at room temperature 


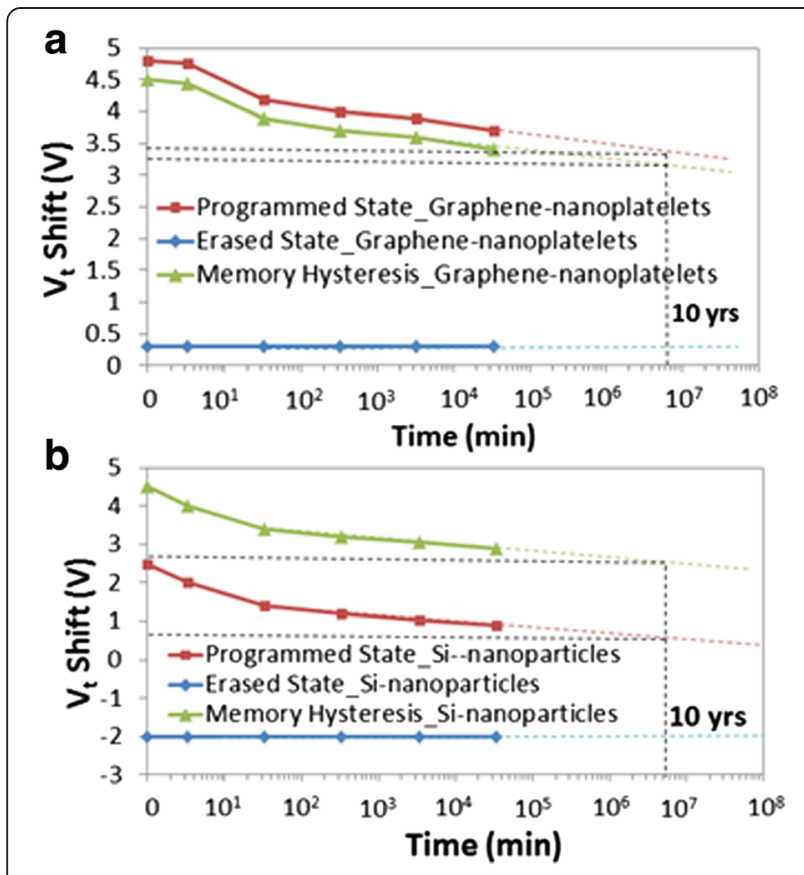

Fig. 3 Memory retention characteristics measured by first programming/ erasing the memory at $8 /-8 \mathrm{~V}$ at room temperature $\mathbf{a}$ with graphene nanoplatelets and $\mathbf{b}$ with Si nanoparticles tunneling (PAT) [17] is the main emission mechanism at $E_{\mathrm{ox}}<5.6 \mathrm{MV} / \mathrm{cm}$. The plot of the natural logarithm of the $V_{\mathrm{t}}$ shift divided by the square of the electric field vs. the reciprocal of the electric field $\left(J=C_{1} E_{\mathrm{ox}^{2}}^{2} e^{-\frac{C_{2}}{E_{\mathrm{ox}}}}\right)$ depicted in Fig. $5 \mathrm{~b}$ shows a linear region at $E_{\mathrm{ox}}>5.6 \mathrm{MeV} / \mathrm{cm}$ confirming that Fowler-Nordheim tunneling [17] becomes dominant at higher electric fields. In this case, electrons tunnel through the $\mathrm{Al}_{2} \mathrm{O}_{3}$ triangular energy barrier and are swept by the electric field into the conduction band of $\mathrm{HfO}_{2}$ then into the conduction band of the graphene nanoplatelets as shown in Fig. 5c.

Also, the larger $\mathrm{CBO}$ between graphene and $\mathrm{Al}_{2} \mathrm{O}_{3}$ compared to the $\mathrm{CBO}$ between $\mathrm{Si}$ nanoparticles and $\mathrm{Al}_{2} \mathrm{O}_{3}$ confirms the enhanced retention with graphene. The trap lifetime of the electrons and holes in the memory devices is calculated by first finding the back-tunneling probability $(T)$ $[17,23]$ :

$$
\mathrm{T}=16 \times\left(\frac{E_{0}}{V_{0}}\right) \times\left(1-\frac{E_{0}}{V_{0}}\right) \times e^{-2 d} \frac{\sqrt{2 m_{0}\left(V_{0}-E_{0}\right)}}{\hbar}
$$

where $V_{0}$ is the potential energy of the barrier, $d$ is the thickness of the barrier, $m_{0}$ is the effective mass in the oxide, and $E_{0}$ is the ground state energy of the electron trapped in a 4.4-nm quantum well (in the case of the graphene nanoplatelets) and is equal to $E_{0}=\frac{\hbar^{2} \pi^{2}}{2 m_{0} L^{2}}$ where $\hbar$ is the reduced Plank's constant and $L$ is the thickness of the storage layer [24-34]. Since in the demonstrated memory devices there are three barriers $\left(\mathrm{HfO}_{2}, \mathrm{Al}_{2} \mathrm{O}_{3}\right.$, and interfacial $\mathrm{SiO}_{2}$ ) that the electron must tunnel

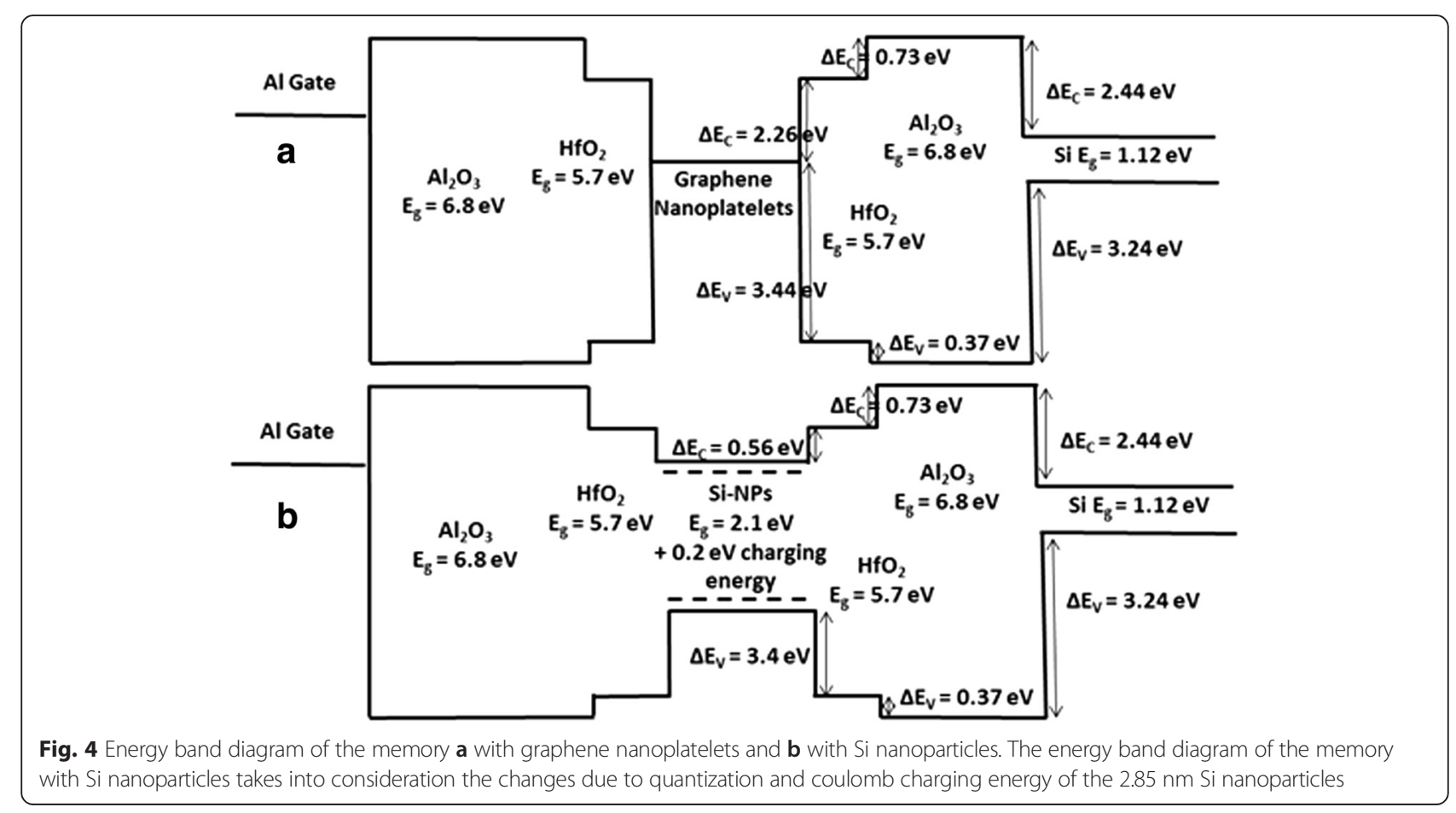



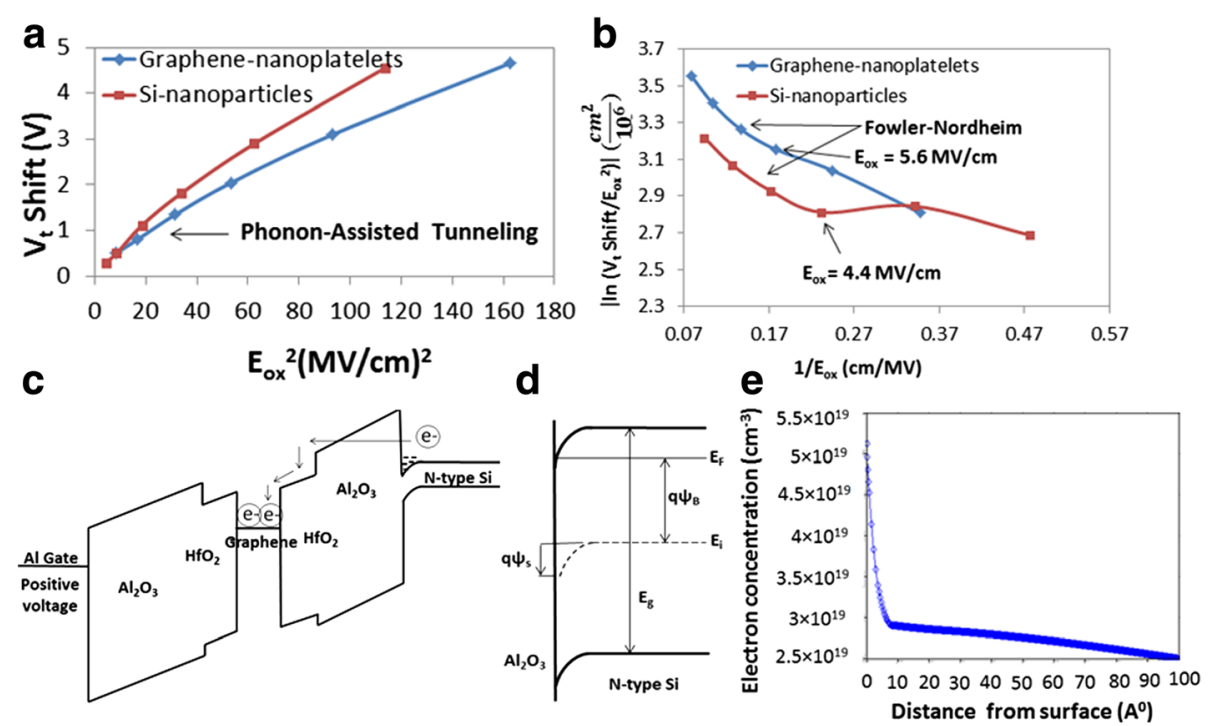

Fig. 5 Charge transport mechanism; a Plot showing the $V_{t}$ shift vs. the square of the electric field across the $\mathrm{Al}_{2} \mathrm{O}_{3}$ for both memories. $\mathbf{b}$ Plot showing the natural logarithm of the $V_{t}$ shift divided by the square of the electric field vs. the reciprocal of the electric field across $\mathrm{Al}_{2} \mathrm{O}_{3}$. $\mathbf{C}$ Energy band diagram of the memory with graphene nanoplatelets under positive gate voltage. $\mathbf{d}$ Energy band diagram near the Si interface of the memory with graphene nanoplatelets. e Plot showing the accumulation electron charge density vs. the distance from the Si interface

through to leak-out, the total transmission probability is thus found by multiplying the transmission probabilities through each oxide and total $T$ is found equal to $\sim 2 \times$ $10^{-23}$ for the memory with graphene nanoplatelets. The electron trap lifetime can be then estimated by $\tau_{\mathrm{e}}=$ $(v T)^{-1}=7.14 \times 10^{8} \mathrm{~s} \sim 23.7$ years where the attempt frequency $v$ in a quantum well [25] is $\frac{E_{0}}{2 \pi \hbar}=7 \times 10^{13} \mathrm{~s}^{-1}$. Similar calculations are performed for the case of the memory with Si nanoparticles, and the electron trap lifetime is found to be $\tau_{\mathrm{e}} \sim 15.7$ years while the holes trap lifetime is $\tau_{\mathrm{h}} \sim 30$ years which is expected to be much larger due to the very large VBO between $\mathrm{Si}$ nanoparticles and $\mathrm{Al}_{2} \mathrm{O}_{3}\left(\Delta E_{\mathrm{V}}=3.81 \mathrm{eV}\right)$. The calculated results support the measured memory retention characteristic.

Furthermore, the program times for both memories are calculated. Since during the program operation, the electron tunnels through $\mathrm{Al}_{2} \mathrm{O}_{3}$ by Fowler-Nordheim tunneling and is swept by the electric field to the charge trapping layer, then the program speed can be found by multiplying the probability of Fowler-Nordheim tunneling through the $\mathrm{Al}_{2} \mathrm{O}_{3}$ layer $\left(T_{\mathrm{FN}}\right)$ by the attempt-toescape frequency $\left(v_{\mathrm{p}}\right) . T_{\mathrm{FN}}$ can be estimated from Eq. (2) $[25,26]$ :

$$
T_{\mathrm{FN}}=e^{-\frac{4 \sqrt{2 m_{0}} f^{\frac{3}{2}}}{3 e E_{\mathrm{ox}}}}
$$

where $\Phi$ is the $\mathrm{CBO}$ between substrate and $\mathrm{Al}_{2} \mathrm{O}_{3}, E_{\text {ox }}$ is the electric field across $\mathrm{Al}_{2} \mathrm{O}_{3}$, and $e$ is the elementary charge. Since during the program operation, there will be band-bending of the $\mathrm{Si}$ substrate near the interface with $\mathrm{Al}_{2} \mathrm{O}_{3}$, a triangular barrier is formed as shown in Fig. $5 \mathrm{c}, \mathrm{d}$, and the attempt-to-escape frequency in a triangular barrier is [26]:

$$
v_{p}=\sqrt{\frac{2 E_{1} q}{m_{0}}} \frac{1}{2 w}
$$

where $E_{1}=2.34 \times\left\{\left[\frac{\left(q E_{0 x} \hbar\right)^{2}}{2 m_{0}}\right]\right\}^{\frac{2}{3}}$ and $w$ is the thickness of the triangular barrier which can be estimated very well by the accumulation region thickness. The electron concentration in the substrate during accumulation is plotted vs. the distance from surface as shown in Fig. 5e. At a program voltage of $8 \mathrm{~V}$, the charge density in the accumulation region can be estimated from [17] $Q=$ $\left(V_{\mathrm{p}}-V_{\mathrm{t}}\right) \times C_{\mathrm{i}}$ where $V_{\mathrm{p}}$ is the program voltage and $C_{\mathrm{i}}$ is the oxide capacitance per unit area. The corresponding volume charge density is $Q_{\text {acc }}=3.05 \times 10^{19} \mathrm{~cm}^{-3}$ with graphene nanoplatelets which corresponds to an accumulation region thickness of $w=6 \mathrm{~A}^{0}$ as shown in Fig. 5e. Therefore, the program time is calculated by dividing the stored charge $Q$ given by $Q=\frac{V_{\mathrm{t}} \text { shift }}{q \times C_{\mathrm{i}}}$ where $C \mathrm{i}$ is the oxide capacitance, by the program speed, and it is found to be equal to $4.1 \mathrm{~ns}$ at $8 \mathrm{~V}$ with graphene nanoplatelets which is much faster than reported non-volatile memory program times in literature (32 ns at $12 \mathrm{~V}$ [34], $100 \mathrm{~ns}$ at $10 \mathrm{~V}$ [35], $1 \mu \mathrm{s}$ at $10 \mathrm{~V}$ [36]). With Si nanoparticles, the time needed for the electrons to tunnel through $\mathrm{Al}_{2} \mathrm{O}_{3}$ is similarly calculated and found $5.6 \mathrm{~ns}$ which is larger than the write time of the memory with graphene nanoplatelets mainly due to the lower electric 
field across the tunnel oxide in the memory with Si nanoparticles. However, in the case of $\mathrm{Si}$ nanoparticles, the time needed to program the memory is found by adding the time needed for the holes to tunnel back to the substrate as well (since mixed charging is observed in this memory) which results in a program time $>>5.6 \mathrm{~ns}$.

\section{Conclusions}

In conclusion, memory devices with Quattro-layer graphene nanoplatelets and 2.85-nm $\mathrm{Si}$ nanoparticles with $\mathrm{Al}_{2} \mathrm{O}_{3} / \mathrm{HfO}_{2}$ tunnel oxide are demonstrated. The results show that graphene nanoplatelets provide a larger charge trapping state density revealed by the larger memory window, enhanced memory endurance due to the pure electrons storage, and enhanced retention due to the larger conduction band offset between storage layer and $\mathrm{Al}_{2} \mathrm{O}_{3}$. Also, the graphene nanoplatelet memory showed a faster program speed compared to Si nanoparticle memory. Finally, the results confirm that band-engineering of both tunnel oxide and charge trapping layer is essential to enhance the memory characteristics. Also, the results highlight that such memory structures have potential in nextgeneration non-volatile memory devices.

\section{Additional file}

Additional file 1: Supplementary information. The supplementa information include an AFM image of the graphene nanoplatelets, a TEM image of the Si nanoparticles, and a high-angle annular dark-field (HAADF) STEM image of the cross-section of the memory with graphene, in addition to calculations of the accumulation charge concentrations.

\section{Competing interests}

The authors declare that they have no competing interests.

\section{Authors' contributions}

NEA and BBT fabricated the memory devices. NEA designed and performed the experiments, analyzed the data, and drafted the manuscript. BBT conducted TEM imaging. AKO, MN, and AN supervised this study. All authors read and approved the final manuscript.

\section{Acknowledgements}

We gratefully acknowledge the financial support for this work provided by the Masdar Institute of Science and Technology. This work was supported in part by the TUBITAK Grants 109E044, 112M004, 112E052, and 113M815.

\section{Author details \\ ${ }^{1}$ Institute Center for Microsystems-iMicro, EECS, Masdar Institute of Science and Technology Abu Dhabi, Abu Dhabi, United Arab Emirates. \\ ${ }^{2}$ UNAM-National Nanotechnology Research Center, Ankara, Turkey. ${ }^{3}$ Institute of Material Science and Nanotechnology, Ankara, Turkey. ${ }^{4}$ Department of Electrical and Electronics Engineering, Bilkent University, 06800 Ankara, Turkey. ${ }^{5}$ Department of Physics, University of Illinois at Urbana Champaign, 1206 W. Green Street, Urbana, IL 61801, USA.}

Received: 5 March 2015 Accepted: 28 May 2015

Published online: 02 June 2015

\section{References}

1. Novoselov KS, Geim AK, Morozov SV, Jiang D, Zhang Y, Dubonos SV, et al. Electric field effect in atomically thin carbon films. Science. 2004;306:666.

2. Schwierz F. Graphene transistors. Nat Nanotechnol. 2010;5:487-96.
3. Wang S, Pu J, Chan DSH, Cho BJ, Loh KP. Wide memory window in graphene oxide charge storage nodes. Appl Phys Lett. 2010;96:143109.

4. Lee JS, Cho J, Lee C, Kim I, Park J, Kim Y-M, et al. Layer-by-layer assembled charge-trap memory devices with adjustable electronic properties. Nat Nanotechnol. 2007;2:790-5.

5. Hanafi HI, Tiwari S, Khan I. Fast and long retention-time nano-crystal memory. IEEE Trans Electron Devices. 1996;43:1553-8.

6. Nayfeh OM, Antoniadis DA, Mantey K, Nayfeh MH. Memory effects in metal-oxide-semiconductor capacitors incorporating dispensed highly monodisperse $1 \mathrm{~nm}$ silicon nanoparticles. Appl Phys Lett. 2007:90:153105.

7. Belomoin G, Therrien J, Smith A, Rao S, Chaieb S, Nayfeh MH. Observation of a magic discrete family of ultrabright Si nanoparticles. Appl Phys Lett. 2002;80:841

8. Lee JJ, Kwong DK. Metal nanocrystal memory with high-K tunneling barrier for improved data retention. IEEE Elect Dev Lett. 2005;52:507.

9. Bertolazzi S, Krasnozhon D, Kis A. Nonvolatile memory cells based on MoS2/ graphene heterostructures. ACS Nano. 2013;7:3246-52.

10. Nayfeh OM. Nonvolatile memory devices with colloidal $1.0 \mathrm{~nm}$ silicon nanoparticles: principle of operation, fabrication, measurements, and analysis. Cambridge, MA: Ph.D dissertation, EECS, MIT; 2009.

11. El-Atab N, Ozcan A, Alkis S, Okyay AK, Nayfeh A. Low power zinc-oxide based charge trapping memory with embedded silicon nanoparticles via poole-frenkel hole emission. Appl Phys Lett. 2014;104:013112.

12. Yu YJ, Zhao Y, Ryu S, Brus LE, Kim KS, Kim P. Tuning the graphene work function by electric field effect. Nano Lett. 2009;9:3430-4.

13. El-Atab N, Rizk A, Okyay AK, Nayfeh A. Zinc-oxide charge trapping memory cell with ultra-thin chromium-oxide trapping layer. AIP Adv. 2013;3:112116.

14. Datta SS, Strachan DR, Mele EJ, Johnson ATC. Surface potentials and layer charge distributions in few-layer graphene films. Nano Lett. 2009;9:7-11.

15. El-Atab N, Ozcan A, Alkis S, Okyay AK, Nayfeh A. Silicon nanoparticle charge trapping memory cell. Phys Status Solidi (RRL). 2014;8:629. doi:10.1002/ pssr.201409157.

16. El-Atab N, Cimen F, Alkis S, Okyay AK, Nayfeh A. Enhanced memory effect with embedded graphene nanoplatelets in $\mathrm{ZnO}$ charge trapping layer. Appl Phys Lett. 2014;105:033102.

17. Sze SM, Ng KK. Physics of semiconductor devices. 3rd ed. Hoboken, New Jersey: JOHN WILEY \& SONS; 2007.

18. El-Atab N., Rizk A., Tekcan B., Alkis S., Okyay AK., Nayfeh A. Memory effect by charging of ultra-small 2-nm laser-synthesized solution processable Si-nanoparticles embedded in Si-Al2O3-SiO2 structure. Phys. Status Solidi A 2015; 212 / doi:10.1002/pssa.201431802

19. El-Atab N, Ozcan A, Alkis S, Okyay AK, Nayfeh A. 2-nm laser-synthesized Si nanoparticles for low-power charge trapping memory devices. 14th IEEE International Conference on Nanotechnology. 2014. p. 505-9. doi:10.1109/ NANO.2014.6968168.

20. Nayfeh A, Okyay AK, El-Atab N, Ozcan A, Alkis S. Low power zinc-oxide based charge trapping memory with embedded silicon nanoparticles. Invited, 226th ECS Meeting Abstract 2014; no. 46, 2014. p. 2143.

21. Nayfeh A, Okyay AK, El-Atab N, Cimen F, Alkis S. Transparent graphene nanoplatelets for charge storage in memory devices. Invited, 226th ECS Meeting Abstract 2014; no. 37. 2014. p. 1879.

22. El-Atab N, Alqatari S, Souier T, Chiesa M, Okyay A. Diode behavior in ultra-thin low temperature ALD grown zinc-oxide on silicon. AIP Adv. 2013;3:102119.

23. Ganichev SD. Distinction between the Poole-Frenkel and tunneling models of electric-field-stimulated carrier emission from deep levels in semiconductors. Phys Rev. 2000;61:10361.

24. El-Atab N, Cimen F, Alkis S, Ortaç B, Alevli M, Dietz N, et al. Enhanced memory effect via quantum confinement in $16 \mathrm{~nm} \mathrm{InN}$ nanoparticles embedded in ZnO charge trapping layer. Appl Phys Lett. 2014;104:253106.

25. Griffiths DJ. Introduction to quantum mechanics. 2nd ed. New Jersey: Upper Saddle River: Prentice Hall; 2004.

26. Novoselov KS, Geim AK, Morozov SV, Jiang D, Katsnelson MI, Grigorieva IV, et al. Two-dimensional gas of massless Dirac fermions in graphene. Nature. 2005;438:197.

27. Tanner CM, Perng YC, Frewin C, Saddow SE, Chang JP. Electrical performance of Al2O3 gate dielectric films deposited by atomic layer deposition on 4H-SiC. Appl Phys Lett. 2007;91:203510

28. He H, Orlando R, Blanco MA, Pandey R, Amzallag E, Baraille I, et al. Firstprinciples study of the structural, electronic, and optical properties of $\mathrm{Ga}_{2} \mathrm{O}_{3}$ in its monoclinic and hexagonal phases. Phys Rev B. 2006;79:195123. 
29. Li MF, H Yu, YT Hou, J Kang, X Wang, C Shen. Selected topics on $\mathrm{HfO}_{2}$ gate dielectrics for future ULSI CMOS devices. 7th International Conference on Solid-State and Integrated Circuits Technology, Proceedings. 2004;1:366.

30. Lin YS, Puthenkovilakam R, Chang JP. Dielectric property and thermal stability of $\mathrm{HfO}_{2}$ on silicon. Appl Phys Lett. 2002;81:2041.

31. Tsu R, Babić D, loriatti Jr L. Simple model for the dielectric constant of nanoscale silicon particle. J Appl Phys. 1997;82:1327.

32. Takiguchi N, Koba S, Tshuchiya H, Ogawa M. Comparisons of performance potentials of Si and InAs nanowire MOSFETs under ballistic transport. IEEE Trans Electron Devices. 2012;59:206.

33. Mazzeo G, Yablonovitch E, Jiang HW, Bai Y, Fitzgerald EA. Conduction band discontinuity and electron confinement at the $\mathrm{Si}_{x} \mathrm{Ge}_{1-x} / \mathrm{Ge}$ interface. Appl Phys Lett. 2010;96:213501.

34. Choi SJ, Han JH, Kim S, Moon DI, Jang M, Choi YK. A novel TFT with a laterally engineered bandgap for of 3D logic and flash memory. 2010 symposium on VLSI technology digest of technical papers. 2010. p. 111-2.

35. Wang $X$, Kwong DL. A novel high-SONOS memory using TaN/Al2O3/Ta2O5/ $\mathrm{HfO} 2 / \mathrm{Si}$ structure for fast speed and long retention operation. IEEE Trans Electron Devices. 2006:53:78.

36. Wang HX, Liu J, Bai W, Kwong DL. A novel MONOS-type nonvolatile memory using high-k dielectrics for improved data retention and programming speed. IEEE Trans Electron Devices. 2004;51:597-602.

\section{Submit your manuscript to a SpringerOpen ${ }^{\circ}$} journal and benefit from:

- Convenient online submission

- Rigorous peer review

- Immediate publication on acceptance

- Open access: articles freely available online

- High visibility within the field

- Retaining the copyright to your article

Submit your next manuscript at $>$ springeropen.com 